\title{
ENTREVISTA CON ABDALLAH HAMMADI
}

Entrevista electrónica realizada por Isaac Donoso y respondida por Abdallah Hammadi durante el mes de julio del año 2016. Se respeta la expresión y giros propios del autor como muestra de lengua española en Argelia.

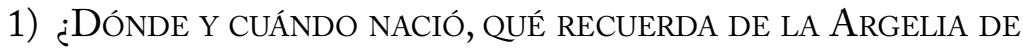
SU INFANCIA?

Nací el 10 de marzo 1947 en una aldea situada al norte de Constantina, casi a 27 kilómetros de la misma, que hoy día lleva el nombre del Héroe de la revolución argelina Zighoud Youcef, nativo de la misma aldea. Mi familia fueron agricultores en aquel pueblo, y nuestro árbol genealógico registrado para la $\mathrm{Ad}-$ ministración colonial francesa existe hasta hoy día.

En los años cincuenta del siglo pasado la colonización francesa confiscó nuestras tierras después de una persecución de muchos hombres de mi familia, considerados rebeldes, hasta el punto que ha obligado a mis padre a refugiarse en Túnez alrededor de los años de 1951. En Túnez nos instalamos en una aldea que se llama Krib Village, departamento de Beja, donde había muchos refugiados argelinos hasta el punto que aquella aldea fue repartida entre argelinos refugiados y tunecinos autóctonos (hablé de aquella convivencia en mi novela titulada Tafunasit editada por la Biblioteca Nacional de Argelia el ano 2004; el titulo Tafunasit es una palabra bereber que significa "la vaca"; en esta novela hablé por primera vez del fenómeno de los refugiados argelinos en la Literatura argelina moderna, y hablé también de mi emigración cultural en España).

En aquel pueblo de campesinos ocurrió mi juventud, y allí empecé mi primer contacto con la escuela primaria colonial antes de la independencia de Túnez, en los años de 1955. En 
aquel pueblo que se llama Krib Village... pueblo completamente campesino, que tiene una feria todos los martes (el zoco) fue donde aprendí mucho del juglar que recita muchas hazañas de la historia arabomusulmana y bereber, hasta llegar a cantar las hazañas de la Revolución Argelina que se proclamó el 1 de noviembre 1954 y duró hasta el 5 de julio 1962.

En aquel pueblo tenía una enseñanza bilingüe árabe y francés. En 1961 llegué a tener el certificado del Sixiéme, y no pude conseguir una beca para ir al Lyceo de la cuidad de Beja por ser argelino. Pero, donde menos esperaba, la revolución argelina conocida con su portavoz el FLN, me ofreció una beca para seguir mis estudios en el Lyceo de la cuidad de Beja en el norte de Túnez, y allí vi por primera vez en mi vida la ciudad, donde todo era diferente del pueblo. Ahí conocí al cine por ejemplo, o los partidos de fútbol, el teatro... En el mes de agosto de 1962 el Gobierno argelino se encargó de devolvernos a nuestra tierra tras la independencia de Argelia, y nos instalamos en la ciudad histórica y mítica de Constantina, que tiene más de tres mil años de existencia y su nombre se debe a su creador, el emperador romano Constantino el Grande.

2) ¿CUÁl FUe SU FORMACIÓn, SUS MAESTROS, CÓMO ERA EL AMBIENTE INTELECTUAL ARGELINO EN SU JUVENTUD?

Mis recuerdos de juventud son de Argelia independiente y de la Argelia del Presidente Houari Boumedien, que encarna la Argelia socialista. En Constantina volví otra vez a la vida de la cuidad, pero esta vez en una cuidad mas grande que la Beja tunecina. Y desde 1962 me llevó mi padre al Lyceo Franco-Musulmán, el único al este de Argelia que aseguraba una formación bilingüe, poco árabe y mucho francés. En el Lyceo tuve la oportunidad de conocer a un profesor libanés cristiano que me dio el curso de filosofía, y estaba casado con una española. Con su presencia con él, en los años 1966 empezó aquella dama tan 
elegante a enseñar la lengua española en aquel Lyceo por primera vez en su historia, y tuve la suerte de estudiar lengua española durante dos años, y de ahí vino mi primer contacto con la lengua española, y por primera vez aprendí el nombre de $\mathrm{Mi}$ guel de Cervantes, autor de la famosa novela Don Quijote de la Mancha, porque durante mi enseñanza francesa nos hizo aprender sólo su historia y su literatura, como si fuese que no existe en el mundo más que Francia.

Después del Lyceo se inauguró en Constantina en los años 1968 la Universidad de Constantina, con tres especialidades solamente: la Medicina en francés hasta hoy día, el Derecho en francés, y Literatura en francés y árabe. Elegí Literatura árabe y, en aquellos años de Nacionalismo y Socialismo, fuimos los literatos de la vanguardia, y tuve la suerte de tener como profesores a unos egipcios, como el gran profesor Ali Abdel-wahid Wafi y Omar Dasuki, y Nabih Hijab y Anas Daud, y otros grandes maestros de la literatura árabe antigua y moderna. Ahí, en aquellas aulas, empecé a escribir mis primeros poemas en los años 1971 y 1972 en un ambiente de lo que llamamos el Realismo social. Por eso me considera la crítica literaria argelina miembro de lo que llaman la "Generación de los años setenta». En mis años universitarios leí mucho los clásicos de la literatura árabe y llegué a aprender de memoria mas de cinco mil versos de poesía árabe clásica. $\mathrm{O}$ sea, tengo una formación clásica que se debe a la influencia directa de mis profesores, que estaban atados a los clásicos, menos mi profesor y poeta Anas Daud, gran especialista de los mitos en la poesía árabe moderna.

En cuanto al ambiente cultural de los setenta del siglo pasado en Argelia, era el Realismo social que domina la vida cultural donde había un grupo de jóvenes progresistas empezaron a escribir la poesía del verso libre y ocuparse de la vida social revolucionaria, según el nuevo proyecto de la sociedad del presidente Houwari Boumedien que se destaca en las tres revolucio- 
nes: revolución agraria, revolución industrial y revolución cultural. Así fue nuestra preocupación, además de ocuparnos con la liberación de los pueblos oprimidos en lo que llamamos el tercer mundo.

3) ¿Por qué FUe A EsTUdiar a EsPaña, DÓNDE Y QUÉ ESTUdió?

Al terminar mis estudios de Literatura en la Universidad de Constantina en 1972, como el mayor en la promoción, la Universidad nos eligió para enseñar en la Facultad de Letras como ayudante entre septiembre de 1972 hasta el 30 de junio de 1973. En aquel tiempo casi no existía ningún profesor argelino que diera clases en la Universidad de Constantina, porque mi generación fue la primera promoción de universitarios argelinos. Tras un año de enseñanza en la universidad, el gobierno de Boumedien decidió preparar nuevos profesores para el futuro de la universidad argelina, y decidió dar becas a casi todos los licenciados, y yo fui el primer estudiante argelino becario que llegó a la España de Franco en septiembre de 1973.

¿El por qué a España? Creo que fue por casualidad, o se debe a aquellas clases de español en el Lyceo Franco-Musulmán de Constantina, porque lo más corriente en Argelia en aquel tiempo era que los estudiantes argelinos siguieran sus estudios superiores en Francia, como era natural durante la colonización francesa. Por eso al llegar a la Embajada de Argelia en Madrid, se quedó estupefacto el agregado cultural, porque no había ningún estudiante argelino en España en aquel tiempo.

Mi experiencia cultural en España fue muy rica, porque al llegar a España tuvo lugar el golpe de Estado en Chile, y la muerte del gran poeta chileno Pablo Neruda, que acababa de publicar su último libro de poesía (El Nexonicidio), y de ahí nació mi gran interés por la poesía española contemporánea y moderna. Por eso terminé mi primera estancia en España con dos 
libros: Introducción a la poesía moderna española y Aproximación al poeta chileno Pablo Neruda.

Mis contactos con la poesía española se deben a mi residencia en el Colegio Mayor de Loyola en Moncloa, en Madrid, donde era el único estudiante árabe en aquella residencia, con una mayoría absoluta de estudiantes españoles de todas las regiones de España, por la cual llegué a conocer las diversidades lingüísticas y culturales de toda España. Además de las actividades culturales que ocurrían en el Colegio Loyola, asistí por primera vez al acto de la pieza teatral del poeta gitano Pepe Heredia Maya (Naquelamos Naquelar), que después de tantos años incluí en mi antología de la poesía andaluza moderna, que publiqué bajo el titulo al-Andalus entre el sueño y la realidad en 2011 en Argelia.

Allí conocí a grandes poetas como Antonio Hernández, Juan Goytisolo, y otros muchos que se encuentran en mi antología, y mi gran recuerdo fue mi visita a casa del gran poeta Vicente Aleixandre, donde fue la ocasión en la que traduje por primera vez algunos de sus poemas al árabe y fueron publicados en la famosa revista iraquí al-Aqlam, y tuve la suerte de recibir de la mano del poeta una dedicatoria de su libro de poesía Sombra del Paraíso.

En cuanto a la Universidad Complutense de Madrid, llegué a tener clases del gran arabista don Emilio García Gómez y también de Fernando de la Granja y del profesor y artista don Elías Terés. Y conocí a mis amigas María Jesús Viguera y María Jesús Rubiera, y a Serafín Fanjul, que tradujo algunos poemas míos, y al gran amigo y gran profesor Pedro Martínez Montávez, que me abrió la revista Almenara, donde he publicado algunos poemas.

Pero lo más destacado en aquellos años de la Universidad Complutense fueron los cursos intensivos de la literatura española contemporánea que daba el gran poeta y gran profesor de 
la crítica literaria modernista Carlos Bousoño, a los que asistí en sus clases durante dos años seguidos y aprendí su teoría innovadora ("el racionalismo verbal en la poesía moderna"), lo que me ha permitido ser uno de los más grandes teóricos de la poesía árabe moderna hoy día.

4) ¿Cuál fue el impacto intelectual Que España dejó en SU FORMACIÓN, Y CUÁLES FUERON SUS MAESTROS ESPAÑOLES?

En España viví la época de Franco, y recuerdo que España era como una isla alejada del mundo. Yo pasé casi un año entero sin encontrar una persona árabe para comunicarme con ella, pero reconozco que España en aquel tiempo era romántica, agradable para vivir, tranquila, y la vida era tan barata que con tres mil pesetas de beca se podía conseguir lo que quisieras. Me recuerdo también de las confrontaciones de los estudiantes con los "grises", como decían en aquel tiempo. Recuerdo también el famoso poema de Rafael Alberti contra Franco: "si los condenas a muerte serán los siete clavos en tu tumba...”.

En el año 1975 me fui a vivir a Granada para terminar mi tesina en Literatura andalusí. Allá conocí al-Andalus y al último reino musulmán de Granada. Pero a mí me gusta más Castilla, y me encanta leer el Poema del Mio Cid y sus encuentros con Al-Mutamid Rey de Sevilla, y con los Almorávides... y su amor por los poetas bandoleros de la época preislámica. Y de allí empecé a interesarme por los famosos romances de frontera, y por la poesía de Garcilaso de la Vega, poeta de la pluma y la espada como al-Mutanabbi.

No puedo negar mi amor por la cultura española y, sobre todo, por sus grandes, como Antonio Machado, García Lorca, Vicente Aleixandre, Luis Cernuda, Pedro Salinas, Miguel Hernández, César Vallejo... y Antonio Gala, etc. Gracias a ellos pude escribir mi famoso diván al-Barzaj wa-Siquin, galardona- 
do para la Fundación al-Babtain en 2002 como «Mejor Diván de la Poesía Árabe Contemporánea».

En Granada tenía como profesores al padre Darío Cabanelas, gran humanista, que me acogió con una fraternidad inolvidable, además de gran conocedor de la literatura andalusí. En cuanto a la vida cultural en Granada, tenía la suerte de conocer al poeta granadino, amigo de todos, Juan de Loxa, que me hizo conocer a todo el círculo literario de Andalucía en general y de Granada en especial, y tuve la suerte de asistir al primer homenaje dedicado a Lorca después de la muerte de Franco. En Granada conocí al gran poeta Rafael Guillén, que se encuentra en mi antología de la poesía andaluza.

Mi segunda estancia en España fue entre los años 1978 y 1980, donde acabé mi tesis doctoral con mi profesor Fernando de la Granja, al que le debo mucho, porque gracias a su generosa labor pude conseguir una beca del Instituto Hispano-Árabe de entonces y seguir en la Complutense. Pero por problemas de salud de mi profesor, don Fernando encargó a la profesora María Jesús Rubiera Mata para seguir conmigo, por ser especialista en la época nazarí de Granada.

Pero mis grandes recuerdos en aquella época fue mi amistad con el gran poeta iraquí Abdelwahab al-Bayati. Esta amistad me hizo ocuparme más de la poesía española, y llegamos a establecer muchas amistades con poetas españoles, ya que tuve que ser el intermediario entre al-Bayati y los poetas españoles. Tuve la suerte de que el día 16 de junio de 1980, fecha de la defensa de mi tesis doctoral, asistió al-Bayati, y el cantaor andaluz Carlos Cano, y mi amigo el poeta Julio Vélez...

5) ¿Por QUÉ DeCidió EsCRIBIR literatura EN LENGUA ESPANOLA?

Por todo lo que acabo de decir, y más todavía, por amor a España, tierra de entrecruce cultural y humano, tierra de mis antepa- 
sados bereberes y árabes, y por ser originario de los hammadíes, facción de los ziríes, reyes de Granada en la época de las Taifas. Me siento ligado a España, por eso amo su cultura, y su arte, y su poesía y su idioma, que tiene mucho de olor árabe, y en toda mi trayectoria cultural sigo buscando aquellos lazos que nos unen como pueblos civilizados que se han aprovechado de la tolerancia y la convivencia a lo largo de los siglos. El Magreb y España tienen una historia casi común, por eso siento los latidos poéticos de Antonio Machado y de García Lorca como si fuesen Nizar Kabbani o Mohammed Laid Califa.

También siento que la lengua española me ha liberado de la lengua colonial francesa, que nos ha atado a una época oscura de opresión e inigualdad, donde la ley colonial de los indígenas nos ha obligado, con fuerza, a creer en su cultura y su tradición y su modo de vida. Por eso quise escribir en español, y con una lengua hermana, donde Cervantes lleva tantos recuerdos de Argel, ciudad de tolerancia y de convivencia. Nada más hay que echar un vistazo a la literatura del cautiverio para confirmar lo que acabo de decir.

6) ¿CUÁl es la ESTÉTICA CON LA QUE TRATÓ DE ESCRIBIR CONVERSO CON EL OLVIDO, Y EL MENSAJE QUE QUERÍA TRANSMITIR?

En cuanto a mi libro de poesía/diván Converso con el olvido, es un canto a una tierra perdida, a una vida de magia y dulzura, a al-Andalus, tierra de arte y de poetas, donde fue el rey Mutamid mecenas de los poetas y amante del cante hondo y de la belleza sublime de la naturaleza y de la humanidad. Converso con el olvido es un amor espiritual a España, tierra de mis amigos del Colegio Loyola, que me obligaron a colaborar con ellos un día para dar a la luz aquel numero de aquella revista que bautizamos humildemente La Buhardilla, y que llevó a cabo publicar mi diván Converso con el olvido como una huella que quedara grabada en el recuerdo. 
La estética de Converso es hispano-árabe-bereber, porque allí se encuentra el Hoggar y el desierto, como se encuentra Castilla y La Mancha y los ecos de Antonio Machado, mi poeta preferido. Es una estética natural, campesina, que hace recordar mi infancia en aquel pueblo lejano durante la emigración forzada. Converso con el olvido es una mano tendida a los poetas españoles.

7) En CUANTO A SU OBRA EN ÁRABE, ¿CUÁL ES LA ESTÉTICA QUE FOMENTA Y CÓMO PUEDE SITUARSE SU PRODUCCIÓN DENTRO DE LA LITERATURA ÁRABE ACTUAL?

Mi obra es variante, primero como poeta argelino y árabe de la generación de los años setenta del siglo veinte. Al principio fui poeta clásico, vista la influencia de mis profesores, y del aprendizaje de memoria de miles de versos de la poesía árabe clásica y, sobre todo, beduina preislámica. Y todo esto culminó con mi teoría poética, que defiende el poema clásico (con métrica y rima) en un manifiesto como prólogo a mi segundo libro de poesías titulado Tahazzaba al-Ichko ya Layla. Este manifiesto quería decir que la innovación del poema clásico no consiste en dejar la métrica y la rima clásica - como dijo la poetisa innovadora, la iraquí Nazik al-Malaika en su teoría-, sino la innovación consiste en crear una lengua nueva y una metáfora irracional. Ésta fue mi teoría en resumen, y de allí hice una llamada a los poetas argelinos para que buscasen en la poesía mística y su experiencia con la lengua árabe, y también tener en cuenta las innovaciones en la poesía europea.

Mi segunda etapa es la etapa europea, que se debe a la influencia directa de la poesía española, influencia poética e influencia de la crítica literaria. La influencia de la crítica literaria me vino de mi maestro Emilio García Gómez en su lectura racional de la poesía árabe en general y andalusí en especial. En cuanto a la poesía modernista, reconozco que el gran teórico y 
poeta Carlos Bousoño me hizo definir de una manera clara y decisiva la diferencia entre la estética antigua y la estética moderna, además de su fabulosa teoría del Racionalismo verbal en la Poesía. Con este entendimiento llegué a escribir mi segunda teoría, que se encuentra como prólogo de mi libro de poesía/diván titulado al-Barzaj wa-Sikkin, libro galardonado para la Fundación del-Babtain como «Mejor Diván de la Poesía Árabe Moderna». Es el único libro de poesía galardonado en Argelia por dicha Fundación, por lo cual recibí una larga carta de felicitación por parte del Presidente Bouteflika en 2002.

Mi tercera etapa es la etapa del "Poema espiritual", que puede encontrarse en mi libro de poesía/diván titulado Antiko an al-hawaa, con un prólogo que define mi nueva visión de lo que es poesía.

8) En el Magreb la literatura marRoduí en lengua espaNOLA TIENE UNA NOTABLE TRADICIÓN, UNA NÓMINA DE AUTORES BASTANTE NUMEROSA Y UNA VITALIDAD CRÍTICA Y LECTORA. EN ARgELIA NO SE ENCUENTRA UN FENÓMENO SIMILAR, A PESAR DE LA VINCULACIÓN CULTURAL, TANTO ANDALUSÍ COMO ESPAÑOLA. ¿SE PUEDE HABLAR DE UNA "LITERATURA HISPANOARGELINA", O "LITERATURA ARGELINA EN LENGUA ESPAÑOLA"? ¿EN QUÉ SITUACIÓN SE ENCUENTRA?

El contacto histórico entre Argelia y España empezó con la llegada del conquistador argelino Táriq ben Ziyad en 711. Este guerrero es de la tribu de Walhasa, de la región de Tremecén del oeste de Argelia, y la gran mayoría de su ejército eran bereberes de las tribus de Sanhaja y Zenata. Desde aquel tiempo fue el primer contacto directo de argelinos con españoles, y después de la llegada de los árabes con Musa ben Nusayr y Buldg ben Bicho, ocurrió la repartición de España entre árabes y bereberes, y según las fuentes fidedignas, la parte sur fue poblada casi para los árabes y la parte norte (Castilla y Aragón, etc.) fue po- 
blada para bereberes argelinos. Digo argelinos porque el ejército del conquistador Táriq es fruto del ejercito del jefe bereber argelino Kusayla al-Awrabi, que asesinó al conquistador árabe Okba ben Nafia cerca de Biskra, y de la Kahina, reina del Aures, que tenía su capital en Tahuda. De allí tuvo el primer contacto que duró ocho siglos de convivencia, y confrontación e intercambio cultural y social, por eso dijo el musicólogo argelino al-Tifachi, natural del pueblo de Tifach cerca de Souk Ahras al este de Argelia, en su obra, que el gran maestro Ibn Bayya del siglo doce hizo una fusión mágica al mezclar música española cristiana con música bereber y árabe, y salió con lo que llamamos hoy día en todo el Magreb "música andalusí" por excelencia, y no fue Ziryab de oriente el verdadero creador de la música andalusí, porque este último no hizo nada más que divulgar la música de su maestro al-Mawsili de Bagdad. Por eso hoy día hay una semejanza entre la música español y la música magrebí.

Este contacto tuvo la suerte de empezar otra vez con la llegada de los españoles a principios del siglo dieciséis (1505), donde fue el segundo contacto desde Orán, que duró 300 anos, de convivencia y confrontación, por eso encontramos hoy día mucha tradición española en la vida de los argelinos del oeste de Argelia. Y durante esta larga estancia pudo Cervantes, según mi lectura propia - que he desarrollado en la introducción de mi libro Vida y obra de Barbarroja-, evocar aquel nombre raro del morisco llamado Sidi Hamete Benenjali, que creo era un personaje verídico que vivió en la época de Barberousse (Keyridin Barbarroja), y fue uno de los descendientes del Reino de Koukou cerca de Argel, y fue un gran jefe aliado de Barbarous. Este personaje mítico es llamado Sidi Hamed Benlkadi, que un tiempo rompió su alianza con Barbarous y pudo echarle de Argel y gobernarla durante cinco años, y los ciudadanos de Argel recuerdan con amargura su dominación después de haber sido el aliado más cercano de Barberous. Y después de treinta años 
de su muerte, llegó Cervantes como cautivo a Argel (16751680), donde la gente seguía recordando las hazañas de Sidi Hamed Benlkadi, que pudo vencer al invencible Barbarroja. Creo que Cervantes encontró en este personaje novelístico lo que buscaba, vencedor del invencible Barbarroja, el enemigo número uno del reino de España y de la cristiandad. Por eso pudo Cervantes mencionar un tal personaje sin ser juzgado por la Inquisición de entonces, y para conmemorar su valentía en Lepanto como buen cristiano. Así hizo también cuando dio la palabra para contar las amarguras de los moriscos a un personaje verídico granadino que se llamaba al-Rakute, a quien le dio la palabra en la novela El Quijote para quejarse en nombre de los oprimidos moriscos. El personaje evocado en El Quijote vivió en tiempos de Alfonso X el Sabio y del rey Muhammad II de Granada, y tenemos muchas noticias sobre este sabio de Ricate en la obra de Ibn al-Jatib. Por eso creo que Sidi Hameti Benenjali es nada mas que Sidi Hamed Benelkadi originario de Bugía y perteneciente a la famosa familia bereber de Ghoubrini.

En Argelia los españoles y los moriscos han dejado muchas huellas en la toponimia de la geografía de Argelia, como el famoso barrio de Argel llamado Taghara que significa tegríes ("los fronterizos").

Después de esta larga presencia llegó la tercera presencia de españoles en Argelia con la colonización francesa durante 1830 hasta 1962, una presencia que siguió también durante la independencia con emigrantes españoles que huyeron del régimen franquista hasta su muerte en 1975. Pero esta presencia fue muy limitada y, sobre todo, durante la época colonial francesa, que fue una colonización cultural y racial, por eso fueron escasos los hispanistas en Argelia. Lo que se cuenta son los becarios argelinos que tuvieron la ocasión de estudiar en España desde los años ochenta del siglo pasado, como Benhamamuch Fátima, 
Kamal Berragda, Abi Ayad, Ismat Turki, Salah Negaoui, Ghawti Kiyat, Bensnousi Ghania...

En cuanto a mi labor de hispanista, al volverme de Madrid en 1980, tuve que dar seminarios como profesor visitante en el departamento de español de la Universidad de Argel y de Orán durante años, y dirigí muchas tesinas y tesis en dichos departamentos. Fueron mis estudiantes de magisterios y doctorados Fatima Ben Hamamouch y Abi-Ayad y Ghania Bensnousi y Salah Negaoui y Ghawti Kiyat y Barbara y otros más...

En Constantina llegué a dar a la luz en la Facultad de Lengua un departamento de español con una iniciativa propia y, después de cuatro años de existencia, y con una promoción de licenciados, hoy día son maestros de español en los liceos. La decisión del Rector de la Universidad de Constantino dio fin al Departamento de español por falta de ayuda de España y falta de lectores de español durante la década negra del terrorismo en Argelia. Así, el rectorado de la Universidad de Constantino cerró el departamento por razón del terrorismo y falta de cooperación con países de habla hispana, y falta de profesores especialistas en español.

9) ¿CUÁles son SUS ACtUales PROYeCtos?

En cuanto a mi labor de traducción, consiste en traducir del español al árabe y del francés al árabe. Dirijo por más de diez años un centro llamado «Laboratorio de traducción en literatura y lingüística». En Argelia la crítica literaria me considera como el primer intelectual que introdujo la literatura española y latinoamericana en Argelia, y el primero que hizo conocer a García Márquez y su realismo mítico en el mundo árabe.

Otro aspecto en mis estudios es la edición crítica de manuscritos y, sobre todo, de obras andalusíes. He editado hasta hoy ocho obras. He escrito obras de literatura árabe en general y literatura argelina antigua y moderna, obras históricas, como la 
Historia del Magreb y de Argelia, siendo la última La Epopeya de la historia de Constantina, que fue estrenada en la inauguración de Constantina como Capital de la cultura árabe el 16 de abril 2015. El último trabajo de traducción que estoy realizando ahora es la traducción de la obra de Martín de Riquer Aproximación al Quijote. 